\title{
Hypothesis for prediction of stimulant drug effectiveness utilizing sensory integrative diagnostic methods
}

JUDITH GIENCKE KIMBALL, PHD, OTR/, FAOTA

Despite extensive research, there has been no way to predict before drug administration which children with attention deficit hyperactivity disorder (ADHD) will respond to medication intended to calm them. A drug trial is the current method used. This paper discusses the action of stimulant medications and presents a hypothesis as to why they work on some children and not others. Sensory integration theory, particularly on vestibular system measures, that involves differential diagnosis of certain types of ADHD children, is used to explain why some children respond to stimulant medications.

D-amphetamine and methylphenidate (Ritalin) long have been used to control attention deficit disorder with hyperactivity (ADHD). Both of these drugs appear to increase attention, although some children respond to one and not the other. More recently, a third stimulant, pemoline (Cylert), has come into use. Its effects on attention are similiar but not identical to the other two drugs.

This varying responsiveness suggests that these drugs do not work by identical mechanisms. There is presently no way to predict how a child will respond to drug therapy for the hyperactivity associated with attention deficit disorder. ${ }^{1-3}$

To date, no diagnostic test battery has been established as a valid predictor of stimulant response in treating ADHD children. Medical practitioners, therefore, have had to rely on the child's response to the drug itself to establish its effectiveness. This procedure often leads to unnecessary problems for children and their parents. It also reflects our general lack of knowledge about drug effects on humans, especially ADHD children.

Reviewing literature on hyperactivity is a difficult task because of the many terms that have been used over the years to describe ADHD children.
The terms minimal brain dysfunction or damage (MBD), hyperactive, and learning disabled (LD) all have been used in studies having subjects that presently would be described as ADHD. Although this paper focuses on ADHD children, the other terms will be used as they appear in the cited literature. Because hyperactivity was not differentiated from the LD and MBD labels, this is the only way to utilize the cited research.

The major question this paper attempts to investigate is: What could be different about the nervous system of the child who responds favorably to stimulant medication to control hyperactivity?

\section{Current theories regarding}

hyperactivity mechanism in ADHD children

Porges $^{4}$ states that research on hyperactivity can be categorized into three physiologic models: (1) hyperactivity affecting "an overaroused or highly aroused CNS;" (2) hyperactivity "as a compensatory behavior to raise the arousal of a suboptimally aroused individual via an increase in proprioceptive sensory input;" or (3) hyperactivity "as a correlate of defective cortical inhibitory mechanisms."

Wender and associates ${ }^{5}$ developed a theory to explain the so-called paradoxic effect of stimulant drugs; that is, the drugs usually used to calm hyperactive children are stimulants. They explain the paradox in terms of a two-component model that includes an excitatory and inhibitory system in the lower brain. They believe that the typical hyperactive child has low cortical norepinephrine levels and, therefore, a deficient inhibitory system, which leads to a state of constant high arousal. Being chemically quite similar to the catecholamines epinephrine and norepinephrine (NE), amphetamines substitute for the NE acting on both the inhibitory and excitatory systems. The net effect for the higharousal children is an increase in inhibitory capacity.

Laufer and colleagues ${ }^{6}$ also take the higharousal position. They think that there is diencephalon dysfunction, specifically, that there is lowered synaptic resistance in the diencephalon, with the 
consequence that incoming stimuli irradiate diffusely and flood the cortex. As a result, these children are overaroused and unable to inhibit or delay responses. Amphetamines increase synaptic resistance, thus reducing the abundance of impulses from the diencephalon.

The opposite conceptual position, low arousal, also has been studied. Satterfield and Dawson ${ }^{7}$ reported that hyperactive children have lower basal skin conduction and less nonspecific galvanic skin response (GSR), which indicates lower autonomic arousal. Overactivity is viewed as secondary to low arousal and serves as a stimulus-generating function. Amphetamines lower activity and calm hyperactive children by raising midbrain reticular activating system (RAS) excitability. Other investigators ${ }^{8}$ have found higher autonomic arousal in hyperactive children, and, in another study, Satterfield and coworkers ${ }^{9}$ found higher skin conduction levels in MBD children.

Satterfield and associates ${ }^{9}$ also found frequent slow dysrhythmias on EEGs of MBD children. They concluded that these children have delayed maturation of the CNS and that good drug responders are low in arousal before treatment. Kornetsky ${ }^{10}$ also believes that hyperactive children are CNS hypoaroused-that there is a dissociation between central and behavioral arousal.

As discussed by Ayres, ${ }^{11}$ differences in autonomic activity may be explained by the differential functioning of the tactile system in MBD and hyperactive children. The hypothesis of raising RAS excitability is demonstrated by her work ${ }^{12,13}$ on postrotary nystagmus.

According to de Quiros and Schrager, ${ }^{14}$ hyperkinesia is externalized by two different symptomshyperactivity and restlessness. Hyperactivity is connected more with brain dysfunction and depends on motor disinhibition elicited by external stimuli. In some of these children, administration of amphetamine or methylphenidate seems to produce a greatly excited state. Restlessness is connected with vestibular-proprioceptive disassociation and depends on postural disinhibition elicited by poor body information (internal stimuli). ${ }^{14}$

Obviously, there are contradictory theories and inconsistent experimental findings. One way out of the dilemma, according to Sroufe, ${ }^{8}$ is to assume that some hyperactive children are overaroused and some underaroused; that is, the variation in arousal level is greater among hyperactive children. He interprets this variation to be contrary to the notion of a syndrome, but, rather, to be indicative of heterogeneity. In order to establish that this variability is due to the existence of two or more types of hyperactive children rather than to sample heterogeneity, consistent predictors of drug response need to be identified.

\section{Differences between good or poor responders to stimulant medication}

Children taking stimulant drugs have long been divided into good and poor response categories by their behavioral manifestations while receiving the medication. More specific differentiation of children by response categories has been done at the Hospital for Sick Children, Toronto, where Swanson and Kinsbourne ${ }^{15}$ have used a paired associate learning procedure (PAL) to evaluate more than 400 children with symptoms of ADHD.

The drug-induced effect on the favorable responders was a rapid decrease in errors on the PAL, which reached its maximum at about two hours after medication. In contrast, the initial druginduced effect on the adverse responders was a rapid increase in errors from baseline, and this deterioration of performance reached its maximum at three hours after medication.

Over many studies, Swanson and colleagues $1,2,15,16$, consistently found that 30 percent of their sample subjects were adverse responders to stimulant medication, even though they carefully selected patients on the basis of ADHD behavior. In their 1978 study, ${ }^{1}$ they stated that these adverse responders best fit the APA subgroup "overanxious," (DSM 308.2), because all children labeled as such were adverse responders. However, some adverse responders were found in other categories. Therefore, all hyperactive children are not the same, although the underlying reason for the differences is not clear.

Swanson and colleagues ${ }^{1}$ concluded that the same symptoms of hyperactivity occur in

at least two quite distinct subpopulations, and that the children in each of the subpopulations respond in an opposite way (author's emphasis added) to administration of stimulant medication (methylphenidate or amphetamine). In this connection, it is noteworthy that overdosed favorable responders yielded time-response curves similar to those of adverse responders. This supports a model that places adverse and favorable responders at opposite ends of a continuum with respect to the psychophysiological concept of CNS arousal or activation level. According to this model, stimulant drugs act to increase CNS arousal level, but this results in a normalizing effect on behavior only for those individuals who have an abnormal condition of underarousal in the unmedicated state (author's emphasis added). Even in those cases, too much drug may push the patient to the other end of the continuum, resulting in "overarousal" and thus losing 
its "normalizing" effect. This suggests quite distinct underlying disorders of brain dysfunction for the two groups of hyperactive children designated as favorable and adverse responders to stimulant medication.

According to Swanson, ${ }^{3}$ knowledge of a patient's medical and behavioral history will not identify favorable responders to stimulants. He concludes that a method is needed to determine hyperactive children who are likely to benefit from stimulant therapy before any drug actually is administered.

\section{Sensory integration}

The research evidence suggests that there is no valid way to distinguish good and poor responders to stimulant drugs beforehand. Drugs are prescribed on the basis of symptoms and are designed to alleviate these symptoms rather than to correct the underlying disorder. No differentiating factors are seen in these children that are predictive of drug response after administration of medication. Swanson, ${ }^{3}$ for example, was unable to detect differences in the hyperactive behavior of good and poor responders. Although predictive distinctions previously have been elusive, there appear to be conceptual reasons for believing that it is possible to develop valid predictive indices.

A set of tests to delineate specific sensory integrative subgroups in children has been developed by Ayres. Her 1965 factor analytic study ${ }^{17}$ correlated 35 tests then commonly used for diagnosing hyperactive, $L D$, and brain-damaged children, with the results identifying five significant factors. Several of her other factor analytic studies, ${ }^{13,18-21}$ confirmed the same set of factors: deficits in postural (vestibular) bilateral integration, praxis (motor planning), form and space perception, auditory language functions, and functions of the left side of the body. Ayre's work in this area led her to develop theoretic constructs and a battery of tests and treatment techniques, which now are commonly referred to as sensory integration or sensory integrative occupational therapy.

The tests designed to delineate the five factors include the Southern California Sensory Integration Tests (SCSIT), the Southern California Postrotary Nystagmus Test (SCPNT), and related clinical observations of $\mathrm{CNS}$ reflex integration. (A new battery of tests, the Sensory Integration and Praxis Tests, currently are under development.)

Of these, the SCPNT is the best single indicator for differentiating between types of sensory integrative problems in LD (including hyperactive) children. ${ }^{13}$ Research ${ }^{12,13,22-24}$ on the SCPNT permits the conclusion that it is a measure unaffected by age or hyperactivity. Therefore, the SCPNT would appear to ba a valid measure to use in differentiating types of hyperactive children.

What exactly does the SCPNT measure? Ayres ${ }^{12}$ has stated that lack of, or short duration of, postrotary nystagmus may be interpreted as overinhibition in the vestibular nuclei. The vestibular nuclei are relay stations for sensory input from the vestibular receptive apparatus in the inner ear. Another hypothesis is that an adequate amount of sensory excitation is not reaching the vestibular nuclei. The nystagmic rhythm is induced in the vestibular nuclei located in the brain stem and relayed to the eyes, thus producing the vestibulo-ocular reflex. Too much neuroinhibition acting on the vestibular nuclei involved in establishing this rhythm could reduce both the duration and excursion of nystagmus: The opposite case, too little neuroinhibition, could result in nystagmus of prolonged duration. Ayres ${ }^{12,13}$ has hypothesized that this prolonged nystagmus or hyperresponsivity may be due to an insufficient amount of inhibition acting on the vestibular nuclei. Other neuroprocesses also may be poorly inhibited.

Ayres ${ }^{13}$ documented the importance of deficits in vestibular functioning in LD (including hyperactive) children; $50 \%$ of a sample of LD children showed depressed vestibular functioning as measured by the SCPNT. Their depressed SCPNT scores improved significantly over matched controls on post-test measures of academic achievement when they were given individual sensory integration therapy to ameliorate their vestibular-balance dysfunction.

Vestibular system dysfunction has been shown ${ }^{19,26,27}$ to lead to form and space perception problems because of the strong basis the vestibular system gives right hemisphere functions. Vestibular system dysfunction and/or tactile system dysfunction also can lead to difficulties in motor planning (dyspraxia). ${ }^{11,13}$

Between 1958 and 1967, deQuiros ${ }^{28}$ performed caloric testing on more than 1,300 newborns in Buenos Aires. Numerous follow-up studies ${ }^{14,29,30}$ on this population revealed that vestibular disorders identified in newborn infants result in later learning problems, and that children with vestibular disorders and related postural disturbances constitute a large segment of the LD population.

In the early experiment, deQuiros ${ }^{29}$ found 68 infants in whom he could identify vestibular deficiency and proprioceptive disturbances at birth. He followed 77 vestibular disabled infants and 83 vestibularly normal infants until they reached the age of 3 years. Differences between the groups indicated the existence of a syndrome in the vestibu- 
lar disabled infant group. The characteristics of this syndrome were vestibular areflexia in response to caloric stimulation, delay of motor development, walking instability, and delayed speech. He then followed this group to age 11, and the differences still were apparent. ${ }^{30}$

In testing primary school children identified as having LD "without apparent justifiable cause," de Quiros ${ }^{29,30}$ found that 52 of 63 had abnormal vestibular responses. He characterized the problems of this group as caloric hyporeflexia, restlessness (hyperactivity), motor problems in reading and writing, and loss of interest in school learning. Also identified were equilibrium problems, low muscle tone, and overimposed emotional disturbance. Duration of nystagmus after rotation is not the only indication of vestibular functioning. Problems in the functioning of the vestibular system also may be reflected by decreased postural reactions, low muscle tone, poor cocontraction, poor one-foot standing balance with vision occluded, gravitational insecurity, or adverse responses to vestibular stimulation, or a combination of these.

The vestibular mechanism has several divisions. The semicircular canals generally are thought to be receptors that detect changes in position (linear movement). The SCPNT is thought to be tapping mainly the semicircular canal response to angular acceleration. Gravitational insecurity is thought to be a utricle-mediated response. If the otoliths of the utricle are displaced, a normal person perceives movement, but gravitationally insecure people feel a fear response as if they were unexpectedly falling. Keeping the utricle in a position of minimum discharge ( 30 degrees forward flexion) is the most comfortable position. ${ }^{31}$

Adverse reactions to the SCPNT may be due to an intravestibular conflict. That is, the semicircular canals and utricle-saccule may be sending conflicting messages concerning the body's position in space, which results in an autonomic nervous system response. A visual-vestibular conflict also is a possible cause.

Beside the vestibular system, the tactile system also is assessed in occupational therapy sensory integrative diagnosis. Some children, including many hyperactive ones, have been shown to have a disordered tactile system. In these children, the protective, excitatory sympathetic dimension of the two-part tactile system (protective-discriminative) stays predominant. The parasympathetic discriminative dimension does not function optimally in its usual inhibitory capacity. As a result, the child interprets tactile stimuli differently than the nor- mal child; in fact, many react as if light-touch stimuli were painful or irritating (the tactile defensive child).

Certain types of tactile stimuli have been found to help to normalize this disordered system. ${ }^{9}$ The differences in autonomic arousal that were noted in several of the drug studies may be related to the tactile system irregularities seen in ADHD children.

Assessment of both the vestibular and tactile system functions is important for determining the type of sensory integrative problem experienced by an ADHD child. The SCSIT, SCPNT, and clinical observations of motor functioning can indicate whether the problem is more likely one of a cortical nature or based on lower center processing problems.

\section{Hypothesis for stimulant drug effectiveness}

The contradictory evidence about the effects of amphetamine, methylphenidate, and pemoline in hyperactive children might be due to the fact that children were not separated according to subgroups. It is now possible to identify subgroups through the use of the SCSIT, the SCPNT, and associated clinical observations. Perhaps the sensory integration differences seen in children are the crucial point in pre-establishing drug effectiveness.

It would appear that the depressed-prolonged postrotary nystagmus dichotomy might provide a theoretic explanation for two types of hyperactivity (similar to the work of Porges ${ }^{4}$ [that is, hyperactivity as a compensatory behavior to raise the arousal of a suboptimally aroused system versus hyperactivity as a result of defective cortical inhibitory mechanisms], and de Quiros and Schrager ${ }^{14}$ [that is, restlessness versus hyperactivity]). It is possible that a child who has an overinhibition of the lower brain area (decreased nystagmus) becomes hyperactive in an attempt to compensate for this inhibition, thus allowing the brain to function more optimally. The effect that fast, excitatory vestibular stimulation has on the hyperactivity in these children is a clue.

Low nystagmus children are calmed by fast vestibular stimulation given during occupational therapy treatment sessions, while high nystagmus children often show increased activity levels. Conversely, perhaps the group of hyperactive children who have decreased cortical inhibition of the lower brain (increased nystagmus) become hyperactive because of this lowered inhibition.

Because methylphenidate and pemoline act primarily as lower brain stimulants, it is possible that 
these drugs release the inhibition of the lower brain in the low nystagmus group, thus allowing them to function more optimally. These children should benefit from a drug therapy regime. The high nystagmus group (those who theoretically have poor cortical inhibition) would only receive more input to their already defective inhibitory system, flooding the cortex with additional signals that could not be properly inhibited, thus increasing hyperactive behavior. Children with this high duration postrotary nystagmus would be expected to be poor responders to stimulant medication.

I previously conducted two studies ${ }^{32,33}$ to investigate the relationship between sensory integrative functioning and response to stimulant medication. Methyphenidate and pemoline were studied (methylphenidate because it long has been the drug of choice for the treatment of hyperactive children, and pemoline because it is a relatively new drug in the field of hyperactivity). Good and poor responders to these two drugs were compared to delineate differences in their sensory integrative profiles; particular attention was paid to vestibular system functioning. In addition, the behaviors of good responders to Ritalin on and off the drug were compared. ${ }^{34}$ Results of the studies did show that good and poor responders to stimulant medication have differing sensory integrative profiles with the primary factor being vestibular system measures.

\section{Conclusions}

These findings confirm the hypothesis that there are at least two distinctly different subgroups in the ADHD population, and suggest that these subgroups need to be identified by researchers involved in studies as well as recognized by those involved in treatment programs. The differentiation of the two groups could lead to substantial differences in research and therapeutic outcomes.

This article is based on a dissertation submitted for partial fulfillment of requirements for a PhD degree, Syracuse University, Syracuse, NY 1980. William Meyers, PhD, Syracuse University, was project consultant; Edward O'Connell, PhD, Syracuse University, was statistical consultant; William Logan, MD, and James Swanson, PhD, Hospital For Sick Children, Toronto, supplied subjects; and Rosalie Sabler-Nadler, OTR, and Margaret Flintoff were unpaid research assistants. This study was supported in part by a grant from the Sensory Integration International, Torrance, Calif.

1. Swanson JS, Kinsbourne M, Roberts W, et al: Time-response analysis of stimulant medication on the learning ability of children referred by hyperactivity. Pediatrics 1978;61:21-29.
2. Kinesbourne M, Swanson J, Herman D: Laboratory measurement of hyperactive childrens' response to stimulant medication in Denoff E, Listern (eds): Minimal Brain Dysfunction: A Development Approach. Masson, NY, 1979.

3. According to a written and phone correspondence with J. Swanson, $\mathrm{PhD}$, (1978).

4. Porges SW: Peripheral and neurochemical parallels of psychopathology: A psychophysiological model relating autonomic imbalance to hyperactivity, psychopathy and autism, in Lipsitt LP, Reese HW (eds): $A d$. vances in Child Development and Behavior. New York, Academic Press, 1976, vol 11, pp 35-65.

5. Wender PH, Epstein RS, Kopin, IJ, et al: Urinary monoamine metabolities in children with minimal brain dysfunction, Am J Psychiatry 1971;127:1411-1415.

6. Laufer MW, Denoff E, Solomons G: Hyperkinetic impulse disorders in children's behavior problems. Psychosom Med 1957;19:38-49.

7. Satterfield JH, Dawson ME: Electrodermal correlates of hyperactivity in children. Psychophysiology 1971;8:191-197.

8. Sroufe LA: Drug treatment of children with behavior problems, in Horowitz FD: Review of Child Development Research. University of Chicago Press, Chicago, 1975, vol 4, pp 347-407.

9. Satterfield JH, Cantwell DP, Lesser LI, et al: Psysiological studies of the hyperkinetic child. Am J Psychiatry 1972;128:1418-1424.

10. Kornetsky C: Minimal brain dysfunction and drugs, in Cruickshank WM, Hallahan DP (eds): Perceptual and Learning Disabilities in Children. Syracuse, NY, Syracuse University Press, 1975.

11. Ayres AJ: Sensory Integration and Learning Disorders. Los Angeles, Western Psychological Services, 1973.

12. Ayres AJ: Southern California Postrotary Nystagmus Test. Los Angeles, Western Psychological Services, 1975.

13. Ayres AJ: The effect of sensory integrative therapy on learning disabled children; (Monograph) Final report of Sensory Integrative Dysfunction, Pasadena, Calif, 1976.

14. de Quiros JB, Schrager OL: Neuropsychological Fundamentals In Learning Disabilities. San Rafael, Calif, Academic Therapy Publications, 1978.

15. Swanson J, Barlow A, Kinsbourne M: Task specificity of responses to stimulant drugs in laboratory tests. Int J Ment Health 1979;8:67-82. 16. Swanson JM, Kinsbourne M: Cognitive effects of stimulant drugs on hyperactive children, in Hale GA, Lewis M (eds): Attention and Cognitive Development. Plenum Publishing Corp, New York, 1979.

17. Ayres AJ: Patterns of perceptual-motor dysfunction in children: A factor analytic study. Percept Mot Skills 1965;20:335-368.

18. Ayres AJ: Interrelations among perceptual-motor abilities in a group of normal children. Am J Occup Ther 1966;20:288-292.

19. Ayres AJ: Interrelationship among perceptual-motor functions in children. Am J Occup Ther 1966;20:68-71.

20. Ayres AJ: Deficits in sensory integration in educationally handicapped children. J Learn Disab 1969;2:160-168.

21. Ayres AJ: Characteristics of types of sensory integrative dysfunction. Am J Occup Ther 1971;25:329-334.

22. Ottenbacher K: Identifying vestibular processing dysfunction in learning disabled children. Am J Occup Ther 1978;32:217-221.

23. Kimball JG: The Southern California Postrotary Nystagmus Test: Stability over time, in Sensory Integration Topics: Faculty Reviews. Pasadena, Calif, Center for the Study of Sensory Integrative Dysfunction, 1980 .

24. Kimball JG: Normative comparison of the Southern California Postrotary Nystagmus Test: Los Angeles vs. Syracuse Data. Am J Occup Ther 1981;35:21-25.

25. Ottenbacher, K: Excessive postrotary nystagmus duration in learning disabled children. Am J Occup Ther 1980;34:40-44.

26. Ayres AJ: Cluster analyses of measures of sensory integration. Am J Occup Ther 1977;31:362-366.

27. Ayres AJ: Learning disabilities and the vestibular system. J Learn Disab 1978;11:88-89.

28. de Quiros JB: Vestibular-proprioceptive integration: Its influence on learning and speech in children. Proceedings of the 10th International American Congress of Psychiatry, Lima, Peru, April 3-7, 1966.

29. de Quiros JB: Diagnosis of vestibular disorders in the learning disabled. J Learn Disab 1976;9:50-58.

30. de Quiros JB: Sensory motor development and language. Conference notes, Center for the Study of Sensory Integrative Dysfunction, Los Angeles, Calif, Sep 29-30, 1979. 
31. According to personal conversation with M. Silberzan (April 1977, Sep 1979).

32. Kimball JG: Prediction of methylphenidate (Ritalin) responsiveness through the use of sensory integrative testing. Am $J$ Occup Therap $1986 ; 40: 241-248$

33. Kimball JG: Prediction of pemoline (Cylert) responsiveness through the use of sensory integrative diagnostics, from a monograph to be published Sensory intergration and attention hyperactivty disorder. Sensory Intergration International, Torrance, Calif.

34. Kimball JG: Differences in performance on sensory integrative measures on and off the stimulant medication Ritalin, from a monograph to be published Sensory intergration and attention hyperactivity disorder. Sensory Integration Internation Torrance, Calif.

From the Division of Occupational Therapy, the University of New England College of Osteopathic Medicine, Biddeford, Me.

Reprint requests to Dr Judith Giencke Kimball, Division of Occupational Therapy, University of New England College of Osteopathic Medicine, Hills Beach Road, Biddeford, Me 04005. 


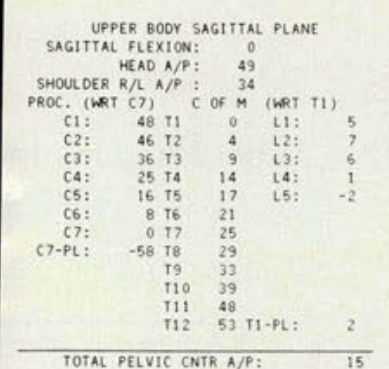

LOWER LIMB RIGHT PELVIC CENTER A/P: PELVIC I/E ROTATION: PELVIC SAGIT. ROTATION TRUE KNEE Y ROTATION: MECH. KNEE Y R ROT. KNEE CENTER A/P: KNEE FLEXION:

PATELLAR HEIGH PATELLAR Q-ANGLE: PATELLAR M/l POSITION:
PATELLAR I/E ROTATION

ANKLE INV/EV ROTATION: ANKLE PL/DOR ROTATION: FOOO ADO FUNCTIONAL LEG LENGTH: ANATOMIC PELVIS/KNEE: ANATOMIC KNEE/ANKLE: ANATOMIC ANKLE/FLOOR:

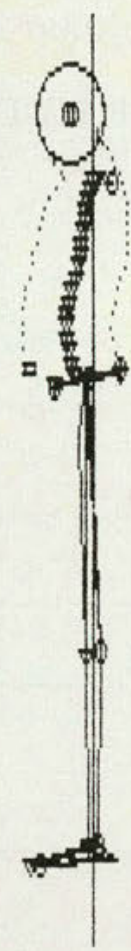

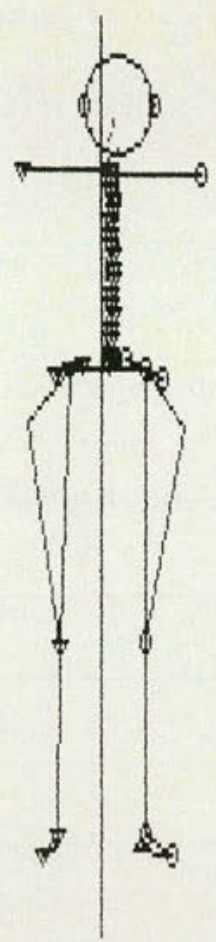

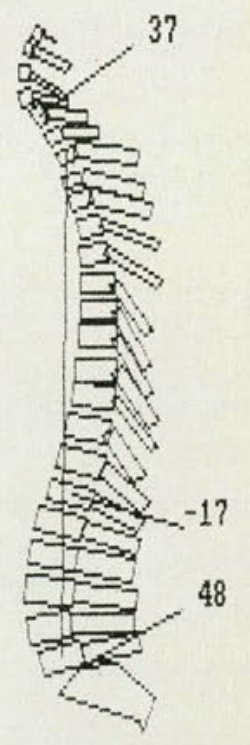

\section{3-Dimensional analysis for manipulative therapy}

and so much more... u'sing

\section{FARO}

METRECOM

Precise, computer-based, non-invasive, 3-dimensional skeletal analysis.

The FARO METRECOM ${ }^{\dagger}$ consists of a

3-dimensional high accuracy digitizer with a PC compatible computer and complete software package. Also included are an instruction manual, videotape and a bibliography of background articles related to the various tests.

Test time varies from 1 to 5 minutes and all information can be stored on diskette for future comparison and/or printed for immediate review. All this for less than $\$ 10,000^{\dagger \dagger}$
Posture and weight distribution

A total body alignment analysis including up to 100 upper and lower body parameters. Optional quadrilateral weight scale measures weight distribution and imbalance.

\section{Spinal intersegmental \& joint flexibility}

Includes forward, lateral bending of the spine, the "flexion" test for sacroiliac function and range of motion in the extremities.

\section{$X$-ray digitization}

Rapid, objective analysis of X-rays including all pelvic, spinal and extremity calculations with the optional x-ray software/hardware package.

\section{FARO MEDICAL TECHNOLOGIES INC. \\ MONTREAL, CANADA WITH OFFICES IN}

NEW YORK, MIAMI, LOS ANGELES, PARIS, MILAN, BERN, MUNICH

$1-(800)-361-6325$ (IN USA)

1-(514)-336-6063 